\title{
Bivariate Generalized Shifted Gegenbauer Orthogonal System
}

\author{
Mohammad A. Alqudah $\mathbb{D}^{1},{ }^{1}$ Maalee N. Almheidat $\mathbb{D}^{2},{ }^{2}$ and Tareq Hamadneh ${ }^{3}$ \\ ${ }^{1}$ German Jordanian University, Amman 11180, Jordan \\ ${ }^{2}$ Department of Mathematics, University of Petra, Amman 11196, Jordan \\ ${ }^{3}$ Department of Mathematics, Al-Zaytoonah University of Jordan, P. O. Box 130, Amman, Jordan
}

Correspondence should be addressed to Maalee N. Almheidat; malmheidat@uop.edu.jo

Received 16 January 2021; Accepted 13 February 2021; Published 1 April 2021

Academic Editor: Kottakkaran Sooppy Nisar

Copyright (c) 2021 Mohammad A. Alqudah et al. This is an open access article distributed under the Creative Commons Attribution License, which permits unrestricted use, distribution, and reproduction in any medium, provided the original work is properly cited.

\begin{abstract}
For $K_{0}, K_{1} \geq 0, \lambda>-(1 / 2)$, we examine $\mathscr{C}_{r}^{*\left(\lambda, K_{0}, K_{1}\right)}(x)$, generalized shifted Gegenbauer orthogonal polynomials, with reference to the weight $W^{\left(\lambda, K_{0}, K_{1}\right)}(x)=\left((2 \lambda \Gamma(2 \lambda)) /\left(\Gamma(\lambda+(1 / 2))^{2}\right)\right)\left(x-x^{2}\right)^{\lambda-(1 / 2)} \square(x \in(0,1)) \mathrm{d} x+K_{0} \delta_{0}+K_{1} \delta_{1}$, where the indicator function is denoted by $\square(x \in(0,1))$ and $\delta_{x}$ indicates the Dirac $\delta$-measure. Then, we construct a bivariate generalized shifted Gegenbauer orthogonal system $\mathfrak{C}_{n, r, d}^{*\left(\lambda, K_{0}, K_{1}\right)}(u, v, w)$ over a triangular domain $T$, with reference to a bivariate measure $W^{\left(\lambda, \gamma, K_{0}, K_{1}\right)}(u, v, w)=\left((\Gamma(2 \lambda+1)) / \Gamma(\lambda+(1 / 2))^{2}\right) u^{\lambda-(1 / 2)}(1-v)^{\lambda-(1 / 2)}(1-w)^{c-1} \square(u \in(0,1-w)) \square(w \in(0,1)) \mathrm{d} u \mathrm{~d} w+K_{0} \delta_{0}(u)$ $+K_{1} \delta_{w-1}(u)$, which is given explicitly in the Bézier form as $\mathfrak{C}_{n, r, d}^{*\left(\lambda, K_{0}, K_{1}\right)}(u, v, w)=\sum_{i+j+k=n} a_{i, j, k}^{n, r, d} \mathbb{B}_{i, j, k}^{n}(u, v, w)$. In addition, for $d=0, \ldots, k, r=0,1, \ldots, n$, and $n \in\{0\} \cup \mathbb{N}$, we write the coefficients $a_{i, j, k}^{n, r, d}$ in closed form and produce an equation that generates the coefficients recursively.
\end{abstract}

\section{Motivation and Introduction}

The Gegenbauer polynomials $C_{k}^{(\lambda)}(x)$ are solutions to the Gegenbauer differential equation $\left(1-x^{2}\right)\left(\left(\mathrm{d}^{2} y\right) / \mathrm{d} x^{2}\right)-(2$ $\lambda+1) x(\mathrm{~d} y / \mathrm{d} x)+k(k+2 \lambda) y=0, k=0,1, \ldots$. They can be written using $P_{k}^{(\alpha, \beta)}(x)$, Jacobi polynomials defined by Szegö [1] where $\beta=\alpha=\lambda-(1 / 2)$ as $C_{k}^{(\lambda)}(x)=(\Gamma(\lambda+(1 / 2))$ $\Gamma(k+2 \lambda) / \Gamma(2 \lambda) \Gamma(k+\lambda+\quad(1 / 2))) P_{k}^{(\lambda-(1 / 2), \lambda-(1 / 2))}(x)$. Even those orthogonal polynomials are described on the interval $[-1,1]$; it is more suitable for analytical and numerical computations to use the interval $[0,1]$.

Finding new orthogonal systems which comprise as eigenfunctions of a differential equation is a motivating task. One of such eigenfunctions was developed by Koornwinder [2] which is the generalized Jacobi polynomials for constants $K_{0}, K_{1} \geq 0$ with parameters $\alpha, \beta \in\{0\} \cup \mathbb{N}$ illustrating orthogonality on the interval $[-1,1]$, where the corresponding differential equation of order $2(\alpha+\beta+3)$ is given to make the corresponding operator widely suitable for applications.

Then, Koekoek and Koekoek [3] established an explicit representation of the generalized Jacobi polynomials, of higher order linear differential equations satisfied by $\left\{P_{k}^{\left(\alpha, \beta, K_{0}, K_{1}\right)}(x)\right\}_{k \in\{0\} \cup \mathbb{N}} ; \alpha, \beta>-1 ; K_{0}, K_{1} \geq 0$. These systems were studied by Koornwinder [2] as orthogonal polynomials with reference to a linear combination of $W^{\left(\alpha, \beta, K_{0}, K_{1}\right)}(x)$, the Jacobi weight, and two delta functions $\delta_{-1}, \delta_{1}$ at the endpoints of the interval $[-1,1]$.

On a square, inducing bivariate generalized orthogonal polynomials is straightforward [4], where tensor product can be utilized. However, the construction over triangular domains is more challenging, where some preliminary abstract was presented in ICNAAM [5].

Now, let $i, j, k$ denote a triple of non-negative integers. In this paper, a construction of orthogonal system of bivariate generalized shifted (BGS) Gegenbauer polynomials in Bézier form over a triangular domain is expressed as $\mathfrak{V}_{n, r, d}^{*\left(\lambda, K_{0}, K_{1}\right)}(u$, $v, w)=\sum_{i+j+k=n} a_{i, j, k}^{n, r, d} \mathbb{B}_{i, j, k}^{n}(u, v, w)$. For $d=0, \ldots, k, r=0,1$, $\ldots, n$, and $n \in\{0\} \cup \mathbb{N}$, we obtain an explicit form for the coefficients $a_{i, j, k}^{n, r, d}$ and produce a recursive formula to calculate the coefficients $a_{i, j, k}^{n, r, d}$ efficiently. 
1.1. Generalized Shifted Gegenbauer Polynomials (GSGP). Generalized orthogonal polynomials were first introduced by Koornwinder [2] and developed by Koekoek and Koekoek in [3]. The univariate shifted Gegenbauer polynomial of degree $j$ in $x$ with parameter $\lambda>-(1 / 2)$ denoted by $C_{j}^{*(\lambda)}(x)$ is achieved by replacing $x$ with $2 x-1$ in the definition of the classical Gegenbauer polynomial, $C_{j}^{(\lambda)}(x)$.

For $K_{0}, K_{1} \geq 0$, the generalized shifted Gegenbauer polynomials (GSGP) of degree $r$ have been characterized by AlQudah in [6] and are given by

$$
\begin{aligned}
& \mathscr{C}_{r}^{*}\left(\lambda, K_{0}, K_{1}\right)(x)=\frac{r !(2 \lambda-1) !}{(r+2 \lambda-1) !} C_{r}^{*(\lambda)}(x) \\
& +\sum_{j=0}^{r} \Lambda_{j} \frac{j !(2 \lambda-1) !}{(j+2 \lambda-1) !} C_{j}^{*(\lambda)}(x) .
\end{aligned}
$$

These polynomials are orthogonal on the interval $[0,1]$ with reference to the weight [2].

$$
\frac{2 \lambda \Gamma(2 \lambda)}{\Gamma(\lambda+(1 / 2))^{2}}\left(x-x^{2}\right)^{\lambda-(1 / 2)} \square(x \in(0,1)) \mathrm{d} x+K_{0} \delta_{0}+K_{1} \delta_{1},
$$

where $\delta_{x}$ is the Dirac $\delta$-measure support at $x$.

$$
\begin{aligned}
\Lambda_{j}= & \left(K_{0}+K_{1}\right) \frac{\Gamma(2 \lambda+j) \Gamma(\lambda+j+(1 / 2))}{(\lambda+(1 / 2)) \Gamma(j) \Gamma(2 \lambda+1) \Gamma(\lambda+j-(1 / 2))} \\
& +K_{0} K_{1} \frac{\Gamma(2 \lambda+j) \Gamma(2 \lambda+j+1)}{(\lambda+(1 / 2))^{2} \Gamma(j)(\Gamma(2 \lambda+1))^{2} \Gamma(j-1)}
\end{aligned}
$$

and $\square(x \in(0,1))$ denotes the indicator function, which is equal to 1 if $x \in(0,1)$, and 0 otherwise.

As the Bernstein polynomials are stable, these polynomials have been expressed in Bernstein form (see [7]). Now, next theorem provides an explicit form for GSGP of degree $r$ in $x$ symbolized by $\mathscr{C}_{r}^{*}\left(\lambda, K_{0}, K_{1}\right)(x)$, written in terms of Bernstein polynomials of degree $r$ in $x$ defined as

$$
\mathbb{B}_{j}^{r}(x)=\frac{r !}{j !(r-j) !} x^{j}(1-x)^{r-j}, \quad j=0,1, \ldots, r .
$$

It is worth mentioning that the polynomials $\mathbb{B}_{j}^{r}(x)$ describe a basis for $\Pi_{r}$, the space of all polynomials of degree $\leq r$. In addition, for integers $i, j, k \geq 0$, we can define the generalized Bernstein polynomials of degree $r$ by $\mathbb{B}_{i j k}^{r}(x, y, z)=((r !) /(i ! j ! k !)) x^{i} y^{j} z^{k}$ where $i+j+k=r$.

Theorem 1 (see [6]). For $\lambda>-(1 / 2), K_{0}, K_{1} \geq 0$, and $\Lambda_{k}$ defined in equation (3), the generalized shifted Gegenbauer polynomials $\mathscr{C}_{r}^{*}\left(\lambda, K_{0}, K_{1}\right)(x)$ of degree $r$ have the following Bernstein representation:

$$
\begin{aligned}
& \mathscr{C}_{r}^{*}\left(\lambda, K_{0}, K_{1}\right)(x)=\frac{r !(2 \lambda-1) !}{(r+2 \lambda-1) !} \sum_{j=0}^{r} \eta_{j, r}^{\lambda} \mathbb{B}_{j}^{r}(x) \\
& +\sum_{k=0}^{r} \frac{\Lambda_{k} k !(2 \lambda-1) !}{(k+2 \lambda-1) !} \sum_{i=0}^{r} \eta_{i, k}^{\lambda} \mathbb{B}_{i}^{k}(x),
\end{aligned}
$$

where

$$
\eta_{j, k}^{\lambda}=(-1)^{k-j} \frac{\left(\begin{array}{c}
k+\lambda-(1 / 2) \\
j
\end{array}\right)\left(\begin{array}{c}
k+\lambda-(1 / 2) \\
k-j
\end{array}\right)}{\left(\begin{array}{l}
k \\
i
\end{array}\right)}
$$

$$
j=0,1, \ldots, k
$$

In addition, Theorem 2 introduced by AlQudah and AlMheidat [8] utilizes the geometric properties of Bernstein basis and proposes the transformation matrix of the GSG basis into Bernstein basis of degree $n$.

Theorem 2 (see [8]). For $r, s=0,1, \ldots, n$ and $\Lambda_{k}$ defined in equation (3), the entries of the transformation matrix of the GS Gegenbauer basis into Bernstein basis of degree $n$ denoted by $\mathbf{A}_{s, r}^{n}$ are defined as

$$
\mathbf{A}_{s, r}^{n}=\mu_{k, s}^{\lambda, n, r}+\sum_{k=0}^{r} \Lambda_{k} \mu_{j, s}^{\lambda, n, k}
$$

where

$$
\mu_{j, s}^{\lambda, n, k}=(k !(2 \lambda-1) !) /\left(\left(\begin{array}{c}
k \\
s
\end{array}\right)(k+2 \lambda-1) !\right)
$$

$\sum_{j=\max (0, s+k-n)}^{\min (s, k)}(-1)^{k-j}\left(\begin{array}{c}n-k \\ s-j\end{array}\right)$ $\left(\begin{array}{c}k+\lambda-(1 / 2) \\ j\end{array}\right)$

$\left(\begin{array}{c}k+\lambda-(1 / 2) \\ k-j\end{array}\right)$.

Since Bézier curves are vital to numerous applications in "approximation theory" and "numerical analysis," with the demand of higher and/or different degree of Bézier curve $[9,10]$, Corollary 1 presented by AlQudah and AlMheidat in [11] uses Bézier degree elevation developed by Farouki and Rajan [10] to write $\mathscr{C}_{j}^{*}\left(\lambda, K_{0}, K_{1}\right)(x)$ of degree $j$ in terms of Bernstein basis of higher and fixed degree $n$, which helps in numerical stability and computation efficiency.

Corollary 1 (see [8]). GS Gegenbauer polynomials $\mathscr{C}_{j}^{*}(\lambda$, $\left.K_{0}, K_{1}\right)(x)$ of degree $j \leq n$ are expressed using Bernstein basis of fixed degree $n$ as

$$
\mathscr{C}_{j}^{*\left(\lambda, K_{0}, K_{1}\right)}(x)=\sum_{s=0}^{n}\left[\mathbf{A}_{s, r}^{n}\right]^{t} \mathbb{B}_{s}^{n}(x), \quad r=0,1, \ldots, n,
$$

where $\left[\mathbf{A}_{s, r}^{n}\right]^{t}$ is the transpose of the matrix $\left[\mathbf{A}_{s, r}^{n}\right]$, for which entries are given in equation (7). 


\section{Bivariate Generalized Shifted Gegenbauer Polynomials on Triangular Domains}

In this section, we introduce an explicit representation of the bivariate generalized shifted (BGS) Gegenbauer polynomials, denoted by $\mathfrak{S}_{n, r, d}^{*\left(\lambda, K_{0}, K_{1}\right)}(u, v, w)$, in terms of Bernstein basis over a triangular domain $T$. With respect to the generalized weight $\mathrm{W}^{\left(\lambda, \gamma, K_{0}, K_{1}\right)}(u, v, w)$, these polynomials create an orthogonal system over $T$.

2.1. The Triangular Domain T. Let $\mathbf{p}_{1}, \mathbf{p}_{2}, \mathbf{p}_{3}$ be noncollinear vertices for a triangle $T$. Then, any point $\mathbf{p}$ inside the triangle can be written uniquely in terms of the vertices $\mathbf{p}_{i}, i=1,2,3$ utilizing a triple of non-negative coordinates, called "barycentric coordinates," defined as $u=\left(\operatorname{area}\left(\mathbf{p}, \mathbf{p}_{2}, \mathbf{p}_{3}\right)\right)$ / $\left(\operatorname{area}\left(\mathbf{p}_{1}, \mathbf{p}_{2}, \mathbf{p}_{3}\right)\right), \quad v=\left(\operatorname{area}\left(\mathbf{p}_{1}, \mathbf{p}, \mathbf{p}_{3}\right)\right) /\left(\operatorname{area}\left(\mathbf{p}_{1}, \mathbf{p}_{2}, \mathbf{p}_{3}\right)\right)$, $w=\left(\operatorname{area}\left(\mathbf{p}_{1}, \mathbf{p}_{2}, \mathbf{p}\right)\right) /\left(\operatorname{area}\left(\mathbf{p}_{1}, \mathbf{p}_{2}, \mathbf{p}_{3}\right)\right)$, such that $u+v=1-$ $w$ and $\mathbf{p}=u \mathbf{p}_{1}+v \mathbf{p}_{2}+w \mathbf{p}_{3}$. We only have two degrees of freedom, despite the fact that we have three "barycentric coordinates."

Thus, any point is exclusively defined by any two of the barycentric coordinates, and thus the triangular domain is defined as $T=\{(u, v, w): u, v, w \geq 0 ; w=1-(u+v)\}$.

For linearly independent and mutually orthogonal polynomials of some degree $n$, employing the barycentric coordinates $(u, v, w)$, we can illustrate the basis in a triangular structure where the $i$ th row contains $i+1$ basis totaling $\left(\begin{array}{c}n+2 \\ 2\end{array}\right)$

It is worthwhile to mention that generalized Bernstein polynomials, $\mathbb{B}_{i j k}^{n}(u, v, w)$, are non-negative and form a partition of unity, $\sum_{0 \leq i, j, k \leq n}(n ! /(i ! j ! k !)) u^{i} v^{j} w^{k}=1$, and $i+j+k=n$

are equally weighted as basis functions with equal integrals over the domain $T$.

Lemma 1 (see [12]). The generalized Bernstein polynomials $\mathbb{B}_{i j k}^{n}(u, v, w)$ such that $i+j+k=n$ on the triangular domain $T$ satisfy $\iint_{T} \mathbb{B}_{i j k}^{n}(u, v, w) d A=\left(\Delta /\left(\begin{array}{c}n+2 \\ 2\end{array}\right)\right), \Delta=2 *$ area $(T)$

2.2. Construction. For $\gamma>-1, n \in\{0\} \cup \mathbb{N} ; k=0, \ldots, n$; $r=0,1, \ldots, n ; d=0,1, \ldots, k ; \Lambda_{k}$ defined by equation (3), $\eta_{i, r}^{\lambda}$ defined by equation (6), where $2 \lambda+\gamma=1$; and analogues to the construction in [13] using barycentric coordinates $u, v, w$, the explicit representation of degree-ordered system of $\mathfrak{C}_{n, r, d}^{*\left(\lambda, K_{0}, K_{1}\right)}(u, v, w)$, BGS Gegenbauer polynomials in Bernstein form over the triangular domain $T$ is written as

$$
\begin{aligned}
& \mathfrak{S}_{n, r, d}^{*\left(\lambda, K_{0}, K_{1}\right)}(u, v, w)=\sum_{i=0}^{r} \eta_{i, r}^{\lambda} \mathbb{B}_{i}^{r}(u, v) \sum_{j=0}^{n-r}(-1)^{j}\left(\begin{array}{c}
n+r+1 \\
j
\end{array}\right) \mathbb{B}_{j}^{n-r}(w, u+v) \\
& +\sum_{k=0}^{n} \Lambda_{k} \sum_{i=0}^{d} \eta_{i, d}^{\lambda} \mathbb{B}_{i}^{d}(u, v) \sum_{j=0}^{k-d}(-1)^{j}\left(\begin{array}{c}
k+d+1 \\
j
\end{array}\right) \mathbb{B}_{j}^{k-d}(w, u+v),
\end{aligned}
$$

these polynomials create an orthogonal system with respect to the weight function $\mathrm{W}^{\left(\lambda, \gamma, K_{0}, K_{1}\right)}(u, v, w)$ given as

$$
\begin{aligned}
& \frac{\Gamma(2 \lambda+1)}{\Gamma(\lambda+(1 / 2))^{2}} u^{\lambda-(1 / 2)}(1-v)^{\lambda-(1 / 2)}(1-w)^{\gamma-1} \llbracket(u \in \\
& \quad(0,1-w)) \rrbracket(w \in(0,1)) \mathrm{d} u \mathrm{~d} w+K_{0} \delta_{0}(u)+K_{1} \delta_{w-1}(u) .
\end{aligned}
$$

The idea of the construction of this system is to let the bivariate $\mathfrak{S}_{n, r, d}^{*\left(\lambda, K_{0}, K_{1}\right)}(u, v, w)$ concur with the univariate shifted Gegenbauer polynomial $C_{n}^{*(\lambda)}(x)$, along one side of $T$, and to make its variation along each chord parallel to that

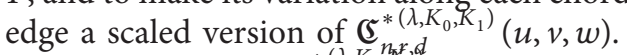

The variation of $\mathfrak{C}_{n, r, d}^{*\left(\lambda, K_{0}, K_{1}, d\right)}(u, v, w)$ with $w$ can be placed in a way that on the triangular domain, these polynomials are orthogonal with every polynomial of degree $<n$, and with other $n$th degree polynomials $\mathfrak{S}_{n, r, d}^{*\left(\lambda, K_{0}, K_{1}\right)}(u, v, w)$ for $r \neq s$.
For $i=0, \ldots, k, k \in\{0\} \cup \mathbb{N}$, employ the polynomial $q_{k, i}(w)$ introduced by Farouki et al. [12] defined as

$$
q_{k, i}(w)=\sum_{j=0}^{k-i}(-1)^{j}\left(\begin{array}{c}
k+i+1 \\
j
\end{array}\right) \mathbb{B}_{j}^{k-i}(w) .
$$

Then, any polynomial $p(w)$ of degree $\leq k-i-1, q_{k, i}(w)$ is orthogonal to $(1-w)^{2 i+l+1}$ on $[0,1]$ for $l=0, \ldots, k-i-1$. Thus,

$$
\int_{0}^{1} q_{k, i}(w)(1-w)^{2 i+1} p(w) \mathrm{d} w=0
$$

By choosing $\mathfrak{C}_{0,0,0}^{*\left(\lambda, K_{0}, K_{1}\right)}=1$, for $0 \leq r \leq n$ and $n=0,1,2, \ldots$, the bivariate polynomials $\mathfrak{C}_{n, r, d}^{*\left(\lambda, K_{0}, K_{1}\right)}(u, v, w)$ form a degree-ordered orthogonal system over the triangular domain $T$.

Using the fact that $(u+v)^{r} \mathbb{B}_{i}^{r}(u /(1-w))=\mathbb{B}_{i}^{r}(u, v)$, we rephrase polynomials (9) as 


$$
\begin{aligned}
\mathfrak{E}_{n, r, d}^{*\left(\lambda, K_{0}, K_{1}\right)}(u, v, w)= & \sum_{i=0}^{r}(-1)^{r-i} \frac{\left(\begin{array}{c}
r+\lambda-(1 / 2) \\
i
\end{array}\right)\left(\begin{array}{c}
r+\lambda-(1 / 2) \\
r-i
\end{array}\right)}{\left(\begin{array}{c}
r \\
i
\end{array}\right)} \mathbb{B}_{i}^{r}\left(\frac{u}{1-w}\right)(1-w)^{r} q_{n, r}(w) \\
& +\sum_{k=0}^{n} \Lambda_{k} \sum_{i=0}^{d}(-1)^{d-i} \frac{\left(\begin{array}{c}
d+\lambda-(1 / 2) \\
i
\end{array}\right)\left(\begin{array}{c}
d+\lambda-(1 / 2) \\
d-i
\end{array}\right)}{\left(\begin{array}{c}
d \\
i
\end{array}\right)} \mathbb{B}_{i}^{d}\left(\frac{u}{1-w}\right)(1-w)^{d} q_{k, d}(w) .
\end{aligned}
$$

Using Theorem 1 , for $d=0, \ldots, k, r=0, \ldots, n$, we get

$$
\begin{aligned}
\mathfrak{S}_{n, r, d}^{*\left(\lambda, K_{0}, K_{1}\right)}(u, v, w)= & C_{r}^{*(\lambda)}\left(\frac{u}{1-w}\right)(1-w)^{r} q_{n, r}(w) \\
& +C_{d}^{*(\lambda)}\left(\frac{u}{1-w}\right)(1-w)^{d} \sum_{k=0}^{n} \Lambda_{k} q_{k, d}(w),
\end{aligned}
$$

where $q_{k, d}(w)$ is defined in equation (11) and $C_{r}^{*(\lambda)}(t)$ denotes the univariate shifted Gegenbauer polynomial of degree $r$.

Two steps are needed to prove that the polynomials $\mathfrak{V}_{n, r, d}^{*\left(\lambda, K_{0}, K_{1}\right)}(u, v, w)$ form an orthogonal system over the triangular domain with respect to the generalized Gegenbauer weight function.

(1) Step 1: we need to show that $\mathfrak{c}_{n, r, d}^{*\left(\lambda, K_{0}, K_{1}\right)}$ $(u, v, w) \in \mathscr{L}_{n}, n \geq 1, r=0,1, \ldots, n$, where for $n \geq 1$, the space $\mathscr{L}_{n}=\left\{p \in \Pi_{n}: p \perp \Pi_{n-1}\right\}$ is defined to be the set of $n$th degree polynomials that are orthogonal to all polynomials of degree $\leq n$ over a triangular domain $T$, which will be given in Theorem 3 .

(2) Step 2: for $r \neq s$ and $d \neq m$, we need to show that $\mathfrak{G}_{n, r, d}^{*\left(\lambda, K_{0}, K_{1}\right)} \perp \mathfrak{G}_{n, s, m}^{*\left(\lambda, K_{0}, K_{1}\right)}$.
Let $r=0, \ldots, n$ and $T$ be a triangular domain; we show that the system of polynomials $\mathfrak{C}_{n, r, d}^{*\left(\lambda, K_{0}, K_{1}\right)}(u, v, w)$ is orthogonal to all polynomials of degree less than $n$ as in the following theorem.

Theorem 3. For $r=0,1, \ldots, n ; n \in \mathbb{N} ; K_{0}, K_{1} \geq 0$; and $\lambda>-(1 / 2), \quad \gamma>-1$, such that $2 \lambda+\gamma=1$, we have $\mathfrak{C}_{n, r, d}^{*\left(\lambda, K_{0}, K_{1}\right)}(u, v, w)$ with respect to the weight function $W^{\left(\lambda, \gamma, K_{0}, K_{1}\right)}(u, v, w)$ written as

$$
\begin{aligned}
& \frac{\Gamma(2 \lambda+1)}{\Gamma(\lambda+(1 / 2))^{2}} u^{\lambda-(1 / 2)}(1-v)^{\lambda-(1 / 2)}(1-w)^{\gamma-1} \square(u \in(0,1-w)) \rrbracket \\
& \cdot(w \in(0,1)) \mathrm{d} u \mathrm{~d} w+K_{0} \delta_{0}(u)+K_{1} \delta_{w-1}(u) .
\end{aligned}
$$

Proof. For $l=0, \ldots, m ; m=0, \ldots, n-1$, construct a set of bivariate polynomials:

$$
H_{l, m}^{(\lambda)}(u, v, w)=C_{l}^{*(\lambda)}\left(\frac{u}{1-w}\right)(1-w)^{m} w^{n-m-1}
$$

where $C_{l}^{*(\lambda)}(t)$ denotes the univariate shifted Gegenbauer polynomial of degree $l$. Employ equation (6); then, $H_{l, m}^{(\chi)}(u, v, w)$ can be expressed as

$$
\begin{aligned}
H_{l, m}^{(\lambda)}(u, v, w) & =(1-w)^{m} w^{n-m-1} \sum_{j=0}^{l}(-1)^{l-j} \frac{\left(\begin{array}{c}
l+\lambda-(1 / 2) \\
j
\end{array}\right)\left(\begin{array}{c}
l+\lambda-(1 / 2) \\
l-j
\end{array}\right)}{\left(\begin{array}{l}
l \\
j
\end{array}\right)} \mathbb{B}_{j}^{l}\left(\frac{u}{1-w}\right) \\
& =(1-w)^{m} w^{n-m-1} \sum_{j=0}^{l} \eta_{j, l}^{\lambda} \mathbb{B}_{j}^{l}\left(\frac{u}{1-w}\right) .
\end{aligned}
$$

Note that span of $H_{l, m}^{(\lambda)}(u, v, w)$ comprises the set of Bernstein polynomials $\mathbb{B}_{j}^{m}(u /(1-w))(1-w)^{m} w^{n-m-1}=$ $\mathbb{B}_{j}^{m}(u, v) w^{n-m-1}, j=0, \ldots, m ; m=0, \ldots, n-1$ spanning $\Pi_{n-1}$. Accordingly, it is sufficient to show that

$$
\begin{aligned}
I= & \iint_{T} \mathfrak{夭}_{n, r, d}^{*\left(\lambda, K_{0}, K_{1}\right)}(u, v, w) H_{l, m}^{(\lambda)}(u, v, w) W^{\left(\lambda, \gamma, K_{0}, K_{1}\right)} \\
& \cdot(u, v, w) \mathrm{d} A=0, \quad l=0, \ldots, m ; m=0, \ldots, n-1 .
\end{aligned}
$$


Now, by substituting $\mathfrak{C}_{n, r, d}^{*\left(\lambda, K_{0}, K_{1}\right)}(u, v, w), H_{l, m}^{(\lambda)}(u, v, w)$, and $W^{\left(\lambda, \gamma, K_{0}, K_{1}\right)}(u, v, w)$ into the integral

$$
\begin{aligned}
I= & \Delta \int_{0}^{1} \int_{0}^{1-w} \mathfrak{夭}_{n, r, d}^{*\left(\lambda, K_{0}, K_{1}\right)}(u, v, w) H_{l, m}^{(\lambda)} \\
& \cdot(u, v, w) W^{\left(\lambda, \gamma, K_{0}, K_{1}\right)}(u, v, w) \mathrm{d} u \mathrm{~d} w,
\end{aligned}
$$

and by collecting, reordering the terms, and applying $t=(u /(1-w))$, we get

$$
\begin{aligned}
\frac{I}{\Delta}= & \frac{\Gamma(2 \lambda+1)}{\Gamma(\lambda+(1 / 2))^{2}} \sum_{j=0}^{\lambda-(1 / 2)}(-1)^{j}\left(\begin{array}{c}
\lambda-(1 / 2) \\
j
\end{array}\right) \int_{0}^{1} C_{r}^{*(\lambda)}(t) C_{l}^{*(\lambda)}(t) t^{\lambda-(1 / 2)}(1-t)^{j} \mathrm{~d} t \int_{0}^{1} w^{n-m-1}(1-w)^{j+\gamma+r+m+\lambda-(1 / 2)} q_{n, r}(w) \mathrm{d} w \\
& +K_{0} \int_{0}^{1} C_{r}^{*(\lambda)}(t) C_{l}^{*(\lambda)}(t) \delta_{0}(t) \mathrm{d} t \int_{0}^{1} w^{n-m-1}(1-w)^{r+m+1} q_{n, r}(w) \mathrm{d} w \\
& +K_{1} \int_{0}^{1} C_{r}^{*(\lambda)}(t) C_{l}^{*(\lambda)}(t) \delta_{1}(t) \mathrm{d} t \int_{0}^{1} w^{n-m-1}(1-w)^{r+m+1} q_{n, r}(w) \mathrm{d} w \\
& +\frac{\Gamma(2 \lambda+1)}{\Gamma(\lambda+(1 / 2))^{2}} \sum_{k=0}^{n} \Lambda_{k} \sum_{j=0}^{\lambda-(1 / 2)}(-1)^{j}(\lambda-(1 / 2) \\
& +(1-w)^{j+\gamma+d+m+\lambda-(1 / 2)} q_{k, d}(w) \mathrm{d} w \\
& +K_{0} \sum_{k=0}^{n} \Lambda_{k} \int_{0}^{1} C_{l}^{*(\lambda)}(t) C_{d}^{*(\lambda)}(t) \delta_{0}(t) \mathrm{d} t \int_{0}^{1}(1-w)^{d+m+1} w^{n-m-1} q_{k, d}^{*}(x) \mathrm{d} w \\
& +K_{1} \sum_{k=0}^{n} \Lambda_{k} \int_{0}^{1} C_{l}^{*(\lambda)}(t) C_{d}^{*}(\lambda) t^{\lambda-(1 / 2)}(1-t)^{j} \mathrm{~d} t \int_{1}(t) \mathrm{d} t \int_{0}^{1}(1-w)^{d+m+1} w^{n-m-1} q_{k, d}(w) \mathrm{d} w .
\end{aligned}
$$

Note that $l<d<r$, when $m<d<r$, and so the orthogonality property of the shifted Gegenbauer polynomials can be used, and when $r \leq d \leq m \leq n-1$, use the orthogonality property given in equation (12). Hence, $I=0$, which completes the proof.

Note that using the generalized weight function $((\Gamma) 2 \lambda+$ $\left.1)) /\left(\Gamma(\lambda+(1 / 2))^{2}\right)\right) u^{\lambda-(1 / 2)}(1-v)^{\lambda-(1 / 2)}(1-w)^{\gamma-1} \square(u \in($ $0,1-w)) \square(w \in(0,1)) \mathrm{d} u \mathrm{~d} w+K_{0} \delta_{0}(u)+K_{1} \delta_{w-1}(u) \quad$ enables us to separate the integrand, and taking the constrained $2 \lambda+\gamma=1$ facilitates the usage of the orthogonality property given in equation (12).

Now we introduce Theorem 4 which will be proved to finalize the construction of the BGS Gegenbauer polynomials orthogonal system over $T,\left\{\mathfrak{夭}_{n, r, d}^{*\left(\lambda, K_{0}, K_{1}\right)}(u, v, w)\right\}$ $\infty, n, n$ $n=0, r=0, d=0$.

Theorem 4. Let $K_{0}, K_{1} \geq 0, \quad \lambda>-(1 / 2), \quad \gamma>-1$, and $W^{\left(\lambda, \gamma, K_{0}, K_{1}\right)}(u, v, w)$ be the generalized weight function defined as $\left((\Gamma(2 \lambda+1)) /\left(\Gamma(\lambda+(1 / 2))^{2}\right)\right) u^{\lambda-(1 / 2)}(1-v)^{\lambda-(1 / 2)}$ $(1-w)^{\gamma-1} \llbracket(u \in(0,1-w)) \square(w \in(0,1)) d u d w+K_{0} \delta_{0}(u)+$ $K_{1} \delta_{w_{\bar{K}} 1}(u)$. Then, for $r \neq s, d \neq m$, we have

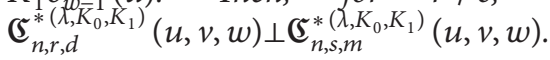

Proof. Let $T=\{(u, v, w): u, v, w \geq 0 ; u+v+w=1\}$ be a triangular domain, and for $r, s=0, \ldots, n ; m=0, \ldots, s$; and $d=0, \ldots, r$, where $r \neq s$ and $d \neq m$, let

$$
\begin{aligned}
I= & \iint_{T} \mathfrak{S}_{n, r, d}^{*\left(\lambda, K_{0}, K_{1}\right)}(u, v, w) \mathfrak{C}_{n, s, m}^{*}\left(\lambda, K_{0}, K_{1}\right) \\
& \cdot(u, v, w) W^{\left(\lambda, \gamma, K_{0}, K_{1}\right)}(u, v, w) \mathrm{d} A,
\end{aligned}
$$

which can be written as

$$
\begin{aligned}
I= & \int_{0}^{1} \int_{0}^{1-w} \mathfrak{夭}_{n, r, d}^{*\left(\lambda, K_{0}, K_{1}\right)}(u, v, w) \mathfrak{C}_{n, s, m}^{*\left(\lambda, K_{0}, K_{1}\right)} \\
& \cdot(u, v, w) W^{\left(\lambda, \gamma, K_{0}, K_{1}\right)}(u, v, w) \Delta \mathrm{d} u \mathrm{~d} w .
\end{aligned}
$$

Note that for $n \in\{0\} \cup \mathbb{N} ; \quad r, k=0, \ldots, n ; \quad$ and $d=0,1, \ldots, k$, the BGS GP defined in equation (14) can be expressed in terms of $q_{n, r}(w)$ as

$$
\begin{aligned}
\mathfrak{夭}_{n, r, d}^{*\left(\lambda, K_{0}, K_{1}\right)}(u, v, w)= & \left(1+\Lambda_{n}\right) C_{r}^{*(\lambda)}\left(\frac{u}{1-w}\right)(1-w)^{r} q_{n, r}(w) \\
& +C_{d}^{*(\lambda)}\left(\frac{u}{1-w}\right)(1-w)^{d} \sum_{k=0}^{n-1} \Lambda_{k} q_{k, d}(w) .
\end{aligned}
$$


By substituting the terms into the integral $I$, making the change of variables $u=t(1-w)$, separating the integrand, and reordering the integrals, the value of $(I / \Delta)$ equals

$$
\begin{aligned}
& \frac{\Gamma(2 \lambda+1)}{\Gamma(\lambda+(1 / 2))^{2}}\left(1+\Lambda_{n}\right)^{2} \sum_{i=0}^{\lambda-(1 / 2)}(-1)^{i}\left(\begin{array}{c}
\lambda-(1 / 2) \\
i
\end{array}\right) \int_{0}^{1} C_{r}^{*(\lambda)}(t) C_{s}^{*(\lambda)}(t)(1-t)^{i} t^{\lambda-(1 / 2)} \mathrm{d} t \\
& \cdot \int_{0}^{1} q_{n, r}(w) q_{n, s}(w)(1-w)^{i+r+s+\gamma+\lambda-(1 / 2)} \mathrm{d} w \\
& +\frac{\Gamma(2 \lambda+1)}{\Gamma(\lambda+(1 / 2))^{2}}\left(1+\Lambda_{n}\right) \sum_{j=0}^{n-1} \Lambda_{j} \sum_{i=0}^{\lambda-(1 / 2)}(-1)^{i}\left(\begin{array}{c}
\lambda-(1 / 2) \\
i
\end{array}\right) \int_{0}^{1} C_{r}^{*(\lambda)}(t) C_{m}^{*(\lambda)}(t)(1-t)^{i} t^{\lambda-(1 / 2)} \mathrm{d} t \\
& \cdot \int_{0}^{1} q_{n, r}(w) q_{j, m}(w)(1-w)^{i+r+m+\gamma+\lambda-(1 / 2)} \mathrm{d} w \\
& +\frac{\Gamma(2 \lambda+1)}{\Gamma(\lambda+(1 / 2))^{2}}\left(1+\Lambda_{n}\right) \sum_{k=0}^{n-1} \Lambda_{k} \sum_{i=0}^{\lambda-(1 / 2)}(-1)^{i}\left(\begin{array}{c}
\lambda-(1 / 2) \\
i
\end{array}\right) \int_{0}^{1} C_{s}^{*(\lambda)}(t) C_{m}^{*(\lambda)}(t)(1-t)^{i} t^{\lambda-(1 / 2)} \mathrm{d} t \\
& \cdot \int_{0}^{1} q_{n, s} q_{n, r}(w) q_{j, m}(w)(1-w)^{i+r+m+\gamma+\lambda-(1 / 2)} \mathrm{d} w \\
& +\frac{\Gamma(2 \lambda+1)}{\Gamma(\lambda+(1 / 2))^{2}} \sum_{k=0}^{n-1} \Lambda_{k} \sum_{j=0}^{n-1} \Lambda_{j} \sum_{i=0}^{\lambda-(1 / 2)}(-1)^{i}\left(\begin{array}{c}
\lambda-(1 / 2) \\
i
\end{array}\right) \int_{0}^{1} C_{d}^{*(\lambda)}(t) C_{m}^{*(\lambda)}(t)(1-t)^{i} t^{\lambda-(1 / 2)} \mathrm{d} t \\
& \cdot \int_{0}^{1} q_{k, d}(w) q_{j, m}(w)(1-w)^{i+d+m+\gamma+\lambda-(1 / 2)} \mathrm{d} w \\
& +K_{0}\left(1+\Lambda_{n}\right)^{2} \int_{0}^{1} C_{r}^{*(\lambda)}(t) C_{s}^{*(\lambda)}(t) \delta_{0}(t) \mathrm{d} t \int_{0}^{1} q_{n, r}(w) q_{n, s}(w)(1-w)^{r+s+1} \mathrm{~d} w \\
& +K_{0}\left(1+\Lambda_{n}\right) \sum_{j=0}^{n-1} \Lambda_{j} \int_{0}^{1} C_{r}^{*(\lambda)}(t) C_{m}^{*(\lambda)}(t) \delta_{0}(t) \mathrm{d} t \int_{0}^{1} q_{n, r}(w) q_{j, m}(w)(1-w)^{r+m+1} \mathrm{~d} w \\
& +K_{0}\left(1+\Lambda_{n}\right) \sum_{k=0}^{n-1} \Lambda_{k} \int_{0}^{1} C_{s}^{*(\lambda)} \int_{0}^{1} q_{n, s}(w) q_{k, d}(w)(1-w)^{s+d+1} \mathrm{~d} w \\
& +K_{0} \sum_{k=0}^{n-1} \Lambda_{k} \sum_{j=0}^{n-1} \Lambda_{j} \int_{0}^{1} C_{d}^{*(\lambda)}(t) C_{m}^{*(\lambda)}(t) \delta_{0}(t) \mathrm{d} t \int_{0}^{1} q_{k, d}(w) q_{j, m}(w)(1-w)^{d+m+1} \mathrm{~d} w \\
& +K_{1}\left(1+\Lambda_{n}\right)^{2} \int_{0}^{1} C_{r}^{*(\lambda)}(t) C_{s}^{*(\lambda)}(t) \delta_{1}(t) \mathrm{d} t \int_{0}^{1} q_{n, r}(w) q_{n, s}(w)(1-w)^{r+s+1} \mathrm{~d} w \\
& +K_{1}\left(1+\Lambda_{n}\right) \sum_{j=0}^{n-1} \Lambda_{j} \int_{0}^{1} C_{r}^{*(\lambda)}(t) C_{m}^{*(\lambda)}(t) \delta_{1}(t) \mathrm{d} t \int_{0}^{1} q_{n, r}(w) q_{j, m}(w)(1-w)^{r+m+1} \mathrm{~d} w \\
& +K_{1}\left(1+\Lambda_{n}\right) \sum_{k=0}^{n-1} \Lambda_{k} \int_{0}^{1} C_{s}^{*(\lambda)}(t) C_{d}^{*(\lambda)}(t) \delta_{1}(t) \mathrm{d} t \int_{0}^{1} q_{n, s}(w) q_{k, d}(w)(1-w)^{s+d+1} \mathrm{~d} w \\
& +K_{1} \sum_{k=0}^{n-1} \Lambda_{k} \sum_{j=0}^{n-1} \Lambda_{j} \int_{0}^{1} C_{d}^{*(\lambda)}(t) C_{m}^{*(\lambda)}(t) \delta_{1}(t) \mathrm{d} t \int_{0}^{1} q_{k, d}(w) q_{j, m}(w)(1-w)^{d+m+1} \mathrm{~d} w .
\end{aligned}
$$


Since $d \neq r$ and $m \neq s$, then $r \neq s$ and $d \neq m$, and so the orthogonality property of the shifted Gegenbauer polynomials is utilized. Then, $I=0$, and thus $\mathfrak{C}_{n, r, d}^{*\left(\lambda, K_{0}, K_{1}\right)}$ $(u, v, w) \perp \mathfrak{C}_{n, s, m}^{*\left(\lambda, K_{0}, K_{1}\right)}(u, v, w)$.

Hence, $\quad$ for $\quad \lambda>-(1 / 2), \quad \gamma>-1, \quad\left\{\mathfrak{夭}_{n, r, d}^{*\left(\lambda, K_{0}, K_{1}\right)}\right.$ $(u, v, w)\}_{n=0, r=0, d=0}^{\infty, n, n}$ form an orthogonal system over the triangular domain $T$, with respect to the generalized weight $W^{\left(\lambda, \gamma, K_{0}, K_{1}\right)}(u, v, w)$.

\section{Recurrence Relation}

Bernstein-Bézier basis has remarkable geometric properties, so they can be used in surfaces and curves which will facilitate numerical computations (see $[9,14])$. For $n, i, j, k \in\{0\} \cup \mathbb{N}$, such that $i+j+k=n$, let the BGS Gegenbauer orthogonal polynomials $\mathfrak{C}_{n, r, d}^{*\left(\lambda, K_{0}, K_{1}\right)}(u, v, w)$, $r=0,1, \ldots, n, d=0, \ldots, k$ be written in terms of Bernstein-Bézier form as follows:

$$
\mathfrak{C}_{n, r, d}^{*\left(\lambda, K_{0}, K_{1}\right)}(u, v, w)=\sum_{i+j+k=n} a_{i, j, k}^{n, r, d} \mathbb{B}_{i, j, k}^{n}(u, v, w) .
$$

Our goal is to obtain a closed formula for coefficients $a_{i, j, k}^{n, r, d}$ and produce a recurrence relation equation to calculate coefficients efficiently.

Theorem 5. For $n \in\{0\} \cup \mathbb{N}, r=0,1, \ldots, n$, and $d=0, \ldots, k$ and non-negative integers $i, j, k$ such that $i+j+k=n$, Bernstein-Bézier coefficients $a_{i, j, k}^{n, r, d}$ of BGS Gegenbauer polynomials $\mathfrak{G}_{n, r, d}^{*\left(\lambda, K_{0}, K_{1}\right)}(u, v, w)=\sum_{i+j+k=n} a_{i, j, k}^{n, r, d} \mathbb{B}_{i, j, k}^{n}(u, v, w)$ are expressed by

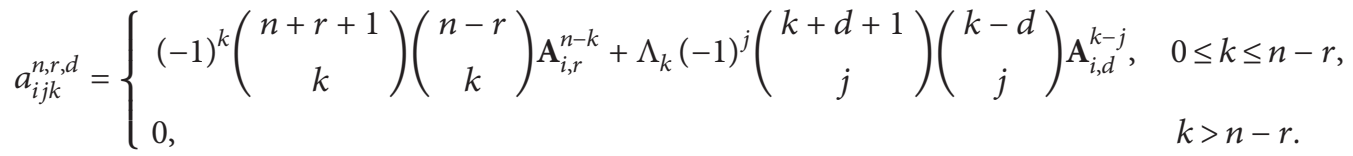

where $\mathbf{A}_{i, r}^{n-k}$ are given in equation (7).

Proof. To find the coefficients $a_{i, j, k}^{n, r, d}$, we have the following cases:

Case 1. $(k>n-r)$. From equation (9), it is obvious that the polynomial $\mathfrak{C}_{n, r, d}^{*\left(\lambda, K_{0}, K_{1}\right)}(u, v, w)$ has degree less than or equal to $n-r$ in the variable $w$. Therefore, $a_{i j k}^{n, r}=0$.
Case 2. $(0 \leq k \leq n-r)$. Equate equation (25) with equation (9), and then we obtain the following:

$$
\begin{aligned}
\sum_{i+j=n-k} a_{i j k}^{n, r} \mathbb{B}_{i j k}^{n}(u, v, w)= & (-1)^{k}\left(\begin{array}{c}
n+r+1 \\
k
\end{array}\right) \mathbb{B}_{k}^{n-r}(w, u+v) \sum_{i=0}^{r} \eta_{i, r}^{\lambda} \mathbb{B}_{i}^{r}(u, v) \\
& +\Lambda_{k} \sum_{j=0}^{k-d}(-1)^{j}\left(\begin{array}{c}
k+d+1 \\
j
\end{array}\right) \mathbb{B}_{j}^{k-d}(w, u+v) \sum_{i=0}^{d} \eta_{i, d}^{\lambda} \mathbb{B}_{i}^{d}(u, v),
\end{aligned}
$$

where $\Lambda_{k}$ is defined in equation (3) and $\eta_{i, r}^{\lambda}$ is defined in equation (6). In the last equation, we compare similar powers of $w$ to obtain

$$
\begin{aligned}
& \sum_{i=0}^{n-k} a_{i j k}^{n, r}\left(\begin{array}{c}
n \\
k
\end{array}\right) \mathbb{B}_{i}^{n-k}(u, v)=\sum_{i=0}^{n-k} a_{i j k}^{n, r} \frac{n !}{i ! j ! k !} u^{i} v^{j}= \\
& (-1)^{k}\left(\begin{array}{c}
n+r+1 \\
k
\end{array}\right)\left(\begin{array}{c}
n-r \\
k
\end{array}\right)(u+v)^{n-r-k} \sum_{i=0}^{r} \eta_{i, r}^{\lambda} \mathbb{B}_{i}^{d}(u, v) \\
& \quad+\Lambda_{k} \sum_{j=0}^{k-d}(-1)^{j}\left(\begin{array}{c}
k+d+1 \\
j
\end{array}\right)\left(\begin{array}{c}
k-d \\
j
\end{array}\right)(u+v)^{k-d-j} \sum_{i=0}^{d} \eta_{i, d}^{\lambda} \mathbb{B}_{i}^{d}(u, v) .
\end{aligned}
$$


Then, we utilize Corollary 1 and some combinatorial identities to write $\sum_{i=0}^{n-k} a_{i j k}^{n, r}\left(\begin{array}{l}n \\ k\end{array}\right) \mathbb{B}_{i}^{n-k}(u, v)$ in above equation as

$$
\begin{aligned}
& (-1)^{k}\left(\begin{array}{c}
n+r+1 \\
k
\end{array}\right)\left(\begin{array}{c}
n-r \\
k
\end{array}\right) \sum_{i=0}^{r} \mathbf{A}_{i, r}^{n-k} \mathbb{B}_{i}^{n-k}(u, v)+\Lambda_{k} \sum_{j=0}^{k-d}(-1)^{j} \\
& \cdot\left(\begin{array}{c}
k+d+1 \\
j
\end{array}\right)\left(\begin{array}{c}
k-d \\
j
\end{array}\right) \sum_{i=0}^{d} \mathbf{A}_{i, d}^{k-j} \mathbb{B}_{i}^{k-j}(u, v) .
\end{aligned}
$$

Therefore, we obtain a closed form for Bernstein-Bézier coefficients.

We will incorporate a result of Farouki et al. [12] to find the recursive formula for $a_{i j k}^{n, r}$, which will be used in the process of obtaining the recurrence equation.

Lemma 2. Let $g(x)=\sum_{i+j+k=m} d_{i, j, k} \mathbb{B}_{i, j, k}^{m}(x) \in \Pi_{m}$. Then, the following holds:

$$
\begin{gathered}
g(x) \in \mathscr{L}_{m} \Leftrightarrow \sum_{i+j+k=m} d_{i, j, k} c_{i, j, k}=0, \\
\forall h(x)=\sum_{i+j+k=m} c_{i, j, k} \mathbb{B}_{i, j, k}^{m}(x) \in \Pi_{m-1} .
\end{gathered}
$$

Now, use $\mathbb{B}_{i j k}^{n-1}(u, v, w)=(u+v+w) \mathbb{B}_{i j k}^{n-1}(u, v, w)$ to express the generalized $(n-1)$ th degree Bernstein polynomial $\mathbb{B}_{i j k}^{n-1}(u, v, w)$ as

$$
\begin{aligned}
& \frac{1}{n}\left[(i+1) \mathbb{B}_{i+1, j, k}^{n}(u, v, w)+(j+1) \mathbb{B}_{i, j+1, k}^{n}(u, v, w)\right. \\
& \left.\quad+(k+1) \mathbb{B}_{i, j, k+1}^{n}(u, v, w)\right] .
\end{aligned}
$$

For $i+j+k=n-1$, using the construction of the BGS Gegenbauer polynomials $\mathfrak{C}_{n, r, d}^{*\left(\lambda, K_{0}, K_{1}\right)}(u, v, w)$, we get $\left\langle\mathbb{B}_{i j k}^{n-1}(u, v, w), \mathfrak{c}_{n, r, d}^{*\left(\lambda, K_{0}, K_{1}\right)}(u, v, w)\right\rangle=0$. Finally, use Lemma 2 to attain the desired recurrence relation to generate the coefficients $a_{i, j, k}^{n, r}$ recursively on $k, a_{i, j, k+1}^{n, r}=(-1 /(k+1))[(i+$ 1) $\left.a_{i+1, j, k}^{n, r}+(j+1) a_{i, j+1, k}^{n, r}\right]$, such that $a_{i, n-i, 0}^{n, r}=\mathbf{A}_{i, r}^{n}$ for $i \in\{0\} \cup \mathbb{N}$ where $\mathbf{A}_{i, r}^{h}$ is given in equation (7).

\section{Applications and Conclusion}

Orthogonal polynomials provide a natural approach to the extension, implementation, and study of solutions to multiple differential equations. They are vital to stability and efficiency of numerical methods, the characteristics of which are useful for solving engineering and physical problems. So, discovering novel types of orthogonal polynomials serve in the advancements of applications in diverse disciplines.

Therefore, orthogonal polynomials have gained notable attention. They are key to numerous problems in mathematical analysis and approximation theory. They play a significant role in splines, Fredholm theory, fractional calculus, integrodifferential equations, stochastic differential equations (SDEs), spectral element method (SEM), and data integration.
It is therefore more practical to construct orthogonal polynomial systems recursively in lieu of absolute forms. The recursive relation makes it possible to establish any polynomial of a given degree directly.

In this paper, we investigated the GS Gegenbauer orthogonal polynomials $\mathscr{C}_{r}^{*}\left(\lambda, K_{0}, K_{1}\right)(x)$ using Bernstein representation on the interval $[0,1]$ with regard to the measure [2] $\quad\left(((2 \lambda) \Gamma(2 \lambda)) / \Gamma(\lambda+(1 / 2))^{2}\right)\left(x-x^{2}\right)^{\lambda-} \quad(1 / 2) \square(x \in$ $(0,1)) \mathrm{d} x+K_{0} \delta_{0}+K_{1} \delta_{1}$.

Then, for $\lambda>-(1 / 2)$, we constructed $\mathfrak{G}_{n, r, d}^{*\left(\lambda, K_{0}, K_{1}\right)}(u, v, w)=\sum_{i+j+k=n} a_{i, j, k}^{n, r, d} \mathbb{B}_{i, j, k}^{n}(u, v, w)$, the BGS Gegenbauer orthogonal polynomials, on the triangular domain $T$ in terms of generalized Bernstein coefficients $a_{i, j, k}^{n, r, d}$, with respect to the weight $((\Gamma(2 \lambda+1)) /(\Gamma(\lambda+$ $\left.\left.(1 / 2))^{2}\right)\right) u^{\lambda-(1 / 2)}(1-v) \quad \lambda^{-(1 / 2)}(1-w)^{\gamma-1} \square(u \in(0,1-w)) \rrbracket$ $(w \in(0,1)) \quad \mathrm{d} u \mathrm{~d} w+K_{0} \delta_{0}(u)+K_{1} \delta_{w-1}(u)$, for $\gamma>-1$, $2 \lambda+\gamma=1$.

We have also shown that $\mathfrak{E}_{n, r, d}^{*\left(\lambda, K_{0}, K_{1}\right)}(u, v, w) \in \mathscr{L}_{n}$, $n \geq 1, r=0, \ldots, n$, and shown that for $r \neq s$ and $d \neq m$,

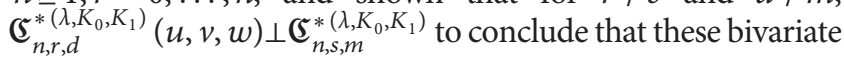
polynomials form an orthogonal system over $T$. The coefficients $a_{i, j, k}^{n, r, d}$ were given in an closed and explicit form, and then a recurrence relationship was defined to allow us to calculate the polynomials effectively and efficiently.

The construction in this paper can be extended to of $i$-variate polynomials over a $i$-dimensional simplex where

$$
\mathbf{T}_{i}=\left\{\mathbf{u}=\left(u_{0}, \ldots, u_{i}\right): u_{j} \geq 0, \sum_{j=0}^{i} u_{j}=1\right\}
$$

denotes the $i$-dimensional unit simplex in barycentric coordinates. Moreover,

$$
\mathbb{B}_{\zeta}^{n}(\mathbf{u})=\left(\begin{array}{c}
n \\
\zeta
\end{array}\right) \mathbf{u}^{\zeta}=\frac{n !}{\zeta_{0} !, \ldots, \zeta_{k} !} u_{0}^{\zeta_{0}} \ldots u_{i}^{\zeta_{i}}, \quad|\zeta|=n,
$$

where $\zeta=\left(\zeta_{0}, \ldots, \zeta_{d}\right) \in\{0\} \cup \mathbb{N}^{i+1}$ and $|\zeta|=\zeta_{0}+\cdots+\zeta_{k}=n$ denotes the Bernstein basis for $n$th degree polynomial over the simplex $\mathbf{T}_{i}$.

\section{Data Availability}

No data were used to support this study.

\section{Conflicts of Interest}

The authors declare that they have no conflicts of interest.

\section{References}

[1] G. Szegö, Orthogonal Polynomials, American Mathematical Society Colloquium Publications, 23, Providence, RI, USA, 4th edition, 1975.

[2] T. H. Koornwinder, "Orthogonal Polynomials with Weight Function $(1-x)^{\alpha}(1+x)^{\beta}+M \delta(x+1)+N \delta(x-1), " C a-$ nadian Mathematical Bulletin, vol. 27, no. 2, pp. 205-214, 1984.

[3] J. Koekoek and R. Koekoek, "Differential equations for generalized Jacobi polynomials," Journal of Computational and Applied Mathematics, vol. 126, no. 1-2, pp. 1-31, 2000. 
[4] P. Suetin, Orthogonal polynomials in two variables, Vol. 3, CRC Press, Boca Raton, FL, USA, 1999.

[5] M. Al-Qudah, M. Al-Mheidat, Y. Al-Jarrah, M. Al-Jarrah, and A. Al-Kazwini, "A recursive relation for generalized Ultraspherical type orthogonal polynomials," in Proceedings of the AIP Conference Proceedings, vol. 1978, no. 1, March 2018, Article ID 050002.

[6] M. A. AlQudah, "Characterization of the generalized gegenbauer polynomials," Applied Mathematical Sciences, vol. 9, no. 102, pp. 5089-5096, 2015.

[7] R. T. Farouki and V. T. Rajan, "On the numerical condition of polynomials in Bernstein form," Computer Aided Geometric Design, vol. 4, no. 3, pp. 191-216, 1987.

[8] M. AlQudah and M. AlMheidat, "Generalized Gegenbauer Koornwinder's type polynomials change of bases," in Proceedings of the AIP Conference Proceedings, vol. 1863, no. 1, May 2017, Article ID 060004.

[9] G. Farin, Curves and Surfaces for Computer-Aided Geometric Design: A Practical Guide, Elsevier, Amsterdam, Netherlands, 2014.

[10] R. T. Farouki and V. T. Rajan, "Algorithms for polynomials in Bernstein form," Computer Aided Geometric Design, vol. 5, no. 1, pp. 1-26, 1988.

[11] M. AlQudah and M. AlMheidat, "Generalized shifted Chebyshev koornwinder's type polynomials: basis transformations," Symmetry, vol. 10, no. 12, p. 692, 2018.

[12] R. T. Farouki, T. N. T. Goodman, and T. Sauer, "Construction of orthogonal bases for polynomials in Bernstein form on triangular and simplex domains," Computer Aided Geometric Design, vol. 20, no. 4, pp. 209-230, 2003.

[13] M. A. AlQudah, "Constrained Ultraspherical-weighted orthogonal polynomials on triangle," International Journal of Mathematical Analysis, vol. 9, no. 2, pp. 61-72, 2015.

[14] J. Hoschek and D. Lasser, Fundamentals of Computer Aided Geometric Design, A. K. Peters, Ltd., Natick, MA, USA, 1993. 